\title{
Conceptual multi-scale dynamic rupture model for the 2011 off the Pacific coast of Tohoku Earthquake
}

\author{
Hideo Aochi ${ }^{1}$ and Satoshi Ide $^{2}$ \\ ${ }^{1}$ Bureau de Recherches Géologiques et Minières, Natural Risks and Safety of CO2 Storage Division, \\ 3 avenue Claude Guillemin, BP36009 Orléans Cedex 2, 45000, France \\ ${ }^{2}$ Graduate School of Science, the University of Tokyo, 1-1-1 Hongo, Bunkyo-ku, Tokyo 113-0033, Japan
}

(Received April 7, 2011; Revised May 7, 2011; Accepted May 11, 2011; Online published September 27, 2011)

\begin{abstract}
We present conceptual dynamic rupture models for the 2011 Tohoku Earthquake based on multi-scale heterogeneity in fracture energy. Regardless of frequently-occurring $M 7.5$ events in this area, it is significant that a large-scale fault heterogeneity corresponding to a $M 9$ event had not been clearly recognized until this earthquake. We show that the largest heterogeneity having a high fracture energy is consistent with the relatively slow rupture propagation of the Tohoku Earthquake. The large gap in fracture energy explains the separation of two groups of waves clearly visible in observed ground motions. Our simulations favour a cascading rupture that begins from a medium heterogeneity and then progresses over larger scale heterogeneity.
\end{abstract}

Key words: 2011 Tohoku Earthquake, multi-scale, dynamic rupture, heterogeneity, ground motion.

\section{Introduction}

The 2011 off the Pacific coast of Tohoku Earthquake, or simply the Tohoku Earthquake, is astonishing in various ways. Speaking seismologically, due to the frequent occurrence of $M \sim 7.5$ events along the Japan Trench in northeastern Japan during the last century, it might have been generally accepted that such a series of events characterize regional fault behavior, without considering the possibility of $M 9$ events. Outside of these modestly-locked sections, or asperities, characterizing $M \sim 7.5$ events, one tends to think that the plate convergence should be relaxed by aseismic slip. However we have learnt that this is not true, although phenomenologically, specifically kinematically, it might not be impossible to model this $M 9$ event using interactive medium-size asperities corresponding to M 7.5 events.

The observed ground motions along the fault strike, namely from north to south on the mainland of Japan (Fig. 1), implies that the rupture propagates mainly to the south (FKS: Fukushima and IBR: Ibaraki) rather than to the north (IWT: Iwate), which is also consistent with the aftershock distribution. Two groups of waves are clearly observed in Miyagi (MYG), and this is also distinguished in the adjacent areas (Iwate and Fukushima), although the waveforms are quite complex. These indicate two distinctive phases in rupture processes around the epicentral region. In the southern end (Ibaraki and CHB: Chiba), the waveform is characterized by a large, rather simple, pulse with a long duration, probably reflecting the effect of rupture directivity or an isolated regional event.

Copyright (c) The Society of Geomagnetism and Earth, Planetary and Space Sciences (SGEPSS); The Seismological Society of Japan; The Volcanological Society of Japan; The Geodetic Society of Japan; The Japanese Society for Planetary Sciences; TERRAPUB.

doi:10.5047/eps.2011.05.008
We have previously proposed multi-scale heterogeneity in fracture energy using circular patches of different sizes on the fault plane. The multi-scalability is necessary to explain the scaling relation between small and large earthquakes and average propagation velocity smaller than the shear-wave velocity (Aochi and Ide, 2004; Ide and Aochi, 2005). Rupture in the existence of such heterogeneity produces a type of cascade growth (Ide and Aochi, 2005) and complexity in seismic cycles (Aochi and Ide, 2009). We show that this is a key concept in understanding the Tohoku Earthquake by presenting dynamic models which start from rupture on a medium patch and which then migrates into a larger patch. Once the rupture develops on a larger scale, smaller heterogeneity no longer plays a principal role in rupture growth. Recently dynamic rupture inversion to obtain frictional or stress parameters has become possible (Peyrat et al., 2004; Di Carli et al., 2010), but the number of parameters is still limited (around 10, i.e. two asperities on a fault plane). Hence, the purpose of this study is not to calibrate the parameters so as to perfectly fit the ground motion observation, but to demonstrate that a conceptual dynamic model can explain the overall behavior of this $M 9$ event.

\section{Models and Numerical Method}

We simply discretize fault heterogeneity using circular patches of different sizes. To account for the fractal nature of faults, the number of patches of radius $r_{n}$ is assumed to follow a power law, $N\left(r_{n}\right) \propto r_{n}^{-2}$. We assume that fracture energy is proportional to patch size and that the stress state is almost homogeneous, which means that the critical slip-weakening distance $D_{\mathrm{c}}$ is scale-dependent adopting a linear slip-weakening friction law with uniform breakdown strength drop (Ide and Aochi, 2005): $D_{\mathrm{c}} \propto r_{n}$. To simplify calculations in this paper, we consider 4 grades of scale 


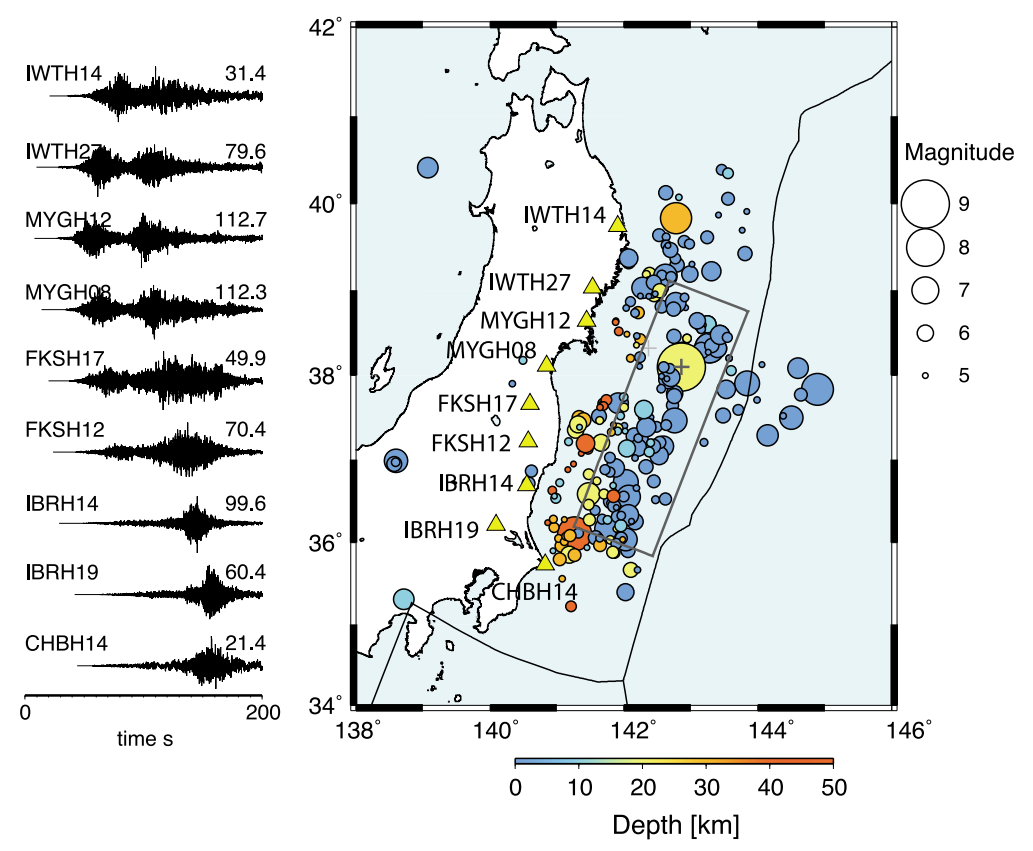

Fig. 1. Map of fault plane projection (black rectangle) and epicenter location (black plus) used in this study based on the reported JMA hypocenter. The grey plus is the epicenter reported by USGS. The seismicity (JMA) is for the period 11th to 15th March 2011. Trench traces are based on information from the University of Texas. The vertical acceleration data at depth (KiK-net by NIED) are shown at selected stations along the whole length of the fault plane and the maximum amplitude is marked in $\mathrm{cm} / \mathrm{s}^{2}$. They are aligned at 14:46:18 (JST).

by taking $r_{1}=100 \mathrm{~km}, r_{2}=50 \mathrm{~km}, r_{3}=25 \mathrm{~km}$, and $r_{4}=12.5 \mathrm{~km}$, with corresponding values of $D_{\mathrm{c}}$ of $1.6,0.8$, 0.4 , and $0.2 \mathrm{~m}$ and the expected number of patches of 1,4 , 16 , and 64, respectively. These numbers of patches are statistically indicative, as the patch density remains arbitrary. What is essential is the existence of the largest patch behind any medium size patches. As there exists a geological limit of the seismogenic zone depth (fault width), it is reasonable to take an ellipse instead of a circle for the largest patch of $r_{1}$. Thus their major and minor axes are $175 \mathrm{~km}$ and $57 \mathrm{~km}$, respectively, for the same surface area.

For modeling spontaneous dynamic rupture processes, we use a 3D boundary integral equation method (BIEM) (Fukuyama and Madariaga, 1995) assuming an infinite, homogeneous elastic medium. Although the rupture most likely broke the free surface near the trench, we keep the model simple and ignore the effect of the free surface. We prepare a volume of $114 \mathrm{~km}$ (parallel to the fault slip and dip directions) and $350 \mathrm{~km}$ (perpendicular to the fault slip and strike directions). For this preliminary computation, we use an element size of $2 \mathrm{~km}$, which is much larger than our usual simulations. A value of $D_{\mathrm{c}}$ less than $40 \mathrm{~cm}$ is not numerically appropriate in this framework. Thus, we assume the minimum $D_{\mathrm{c}}$ as $60 \mathrm{~cm}$ and an initial crack of radius 5-12 km, which cannot represent a true cascade-rupture growth from a very tiny nucleus as shown in Ide and Aochi (2005) and Aochi and Ide (2009). We assume a $P$-wave velocity of $6 \mathrm{~km} / \mathrm{s}$, an $S$-wave velocity of $3.46 \mathrm{~km} / \mathrm{s}$ and a material rigidity of $30 \mathrm{GPa}$. The time step of simulations is $0.167 \mathrm{~s}$. We calculate up to 800 steps (= $133 \mathrm{~s}$ ). We use parallel computing employing MPI-OpenMP (Aochi and Dupros, 2011) to obtain high-performance on multi-core structures.

We impose a breakdown strength drop of $10 \mathrm{MPa}$ as default. Hereafter, our discussion considers only relative stress with respect to an initial shear stress which is taken to be zero, since absolute stress is meaningless under conditions of a planar fault in an infinite medium. We study the variation of patch distributions and strength distribution $\tau_{\mathrm{p}}$ corresponding to stress excess (= strength - initial stress). For later discussions, the projection of the fault plane assumed in this study is geographically shown in Fig. 1, by fixing the hypocenter location at $\left(142.861^{\circ} \mathrm{E}, 38.103^{\circ} \mathrm{N}\right.$, $23.7 \mathrm{~km}$ ) determined by the Japan Meteorological Agency $($ JMA $)$ and the fault plane of (strike, dip $)=\left(201^{\circ}, 9^{\circ}\right)$ after the Global CMT solution. The rake is fixed as $90^{\circ}$.

\section{Parameter Investigation and Results}

Figure 2 shows variations of the dynamic models studied. First of all, we recall that the rupture is not easy to initiate on the largest patch having $D_{\mathrm{c}}=1.6 \mathrm{~m}$, since the elastic system requires a critical crack of a certain dimension. It is possible to investigate theoretically this instability (Shibazaki and Matsu'ura, 1992; Madariaga and Olsen, 2000), but our rapid numerical tests report that a radius of $20 \mathrm{~km}$ is required for $\tau_{\mathrm{p}}=6 \mathrm{MPa}$, as shown in Model A in Fig. 2. This starting process within a diameter of $40 \mathrm{~km}$ might be a quasi-static nucleation and/or dynamic cascade process. It has not been reported to our knowledge that any quasi-static nucleation process occurred just before the mainshock. However, the foreshock of M 7.4 on 9th March 2011 might result in such a nucleus, because the typical size of an $M 7.4$ event is about $50 \mathrm{~km}$. Although the mechanism of the preparation process is an important topic, in this study we are interested only in the dynamic cascade process which can be observed by strong ground motion data.

Figure 3 summarizes the simulations of dynamic rupture propagation for each case shown in Fig. 2. Instead of snap- 

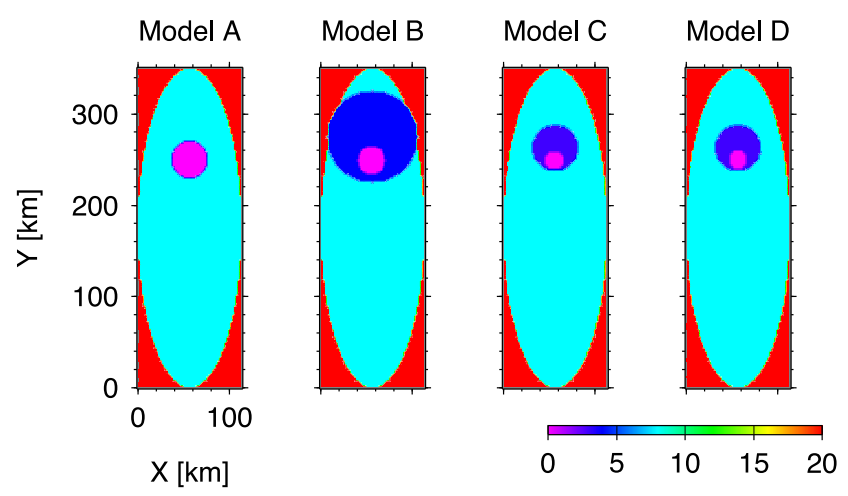

Fracture Energy $\left[\mathrm{MJ} / \mathrm{m}^{2}\right]$
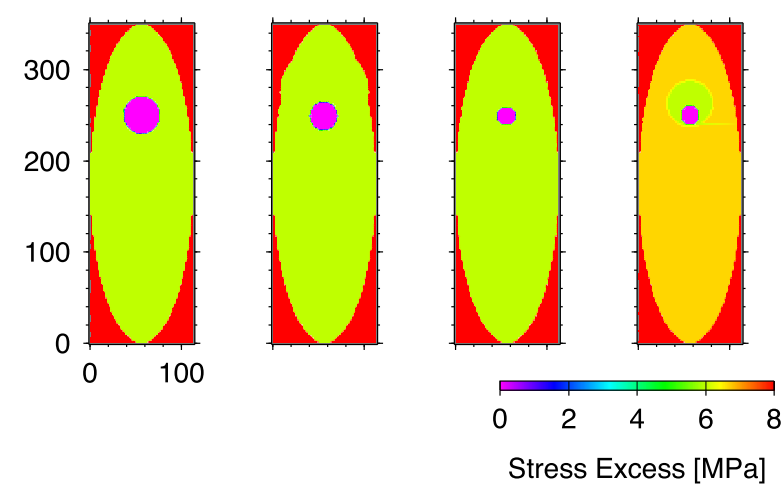

Fig. 2. Conceptual dynamic models based on multi-scale heterogeneous patches: Fracture energy (half of critical slip-weakening distance $\left(D_{\mathrm{c}}\right)$ multiplied by a strength drop in the slip weakening law) in the top panels, and stress excess required for rupture initiation (difference between fault strength $\tau_{\mathrm{p}}$ and initial stress) at the bottom. The area of the smallest fracture energy and zero stress excess corresponds to an initial crack. The largest ellipse patch (1st order) is surrounded by an unbreakable barrier. Model A: Only the 1st order patch. Model B: Combination of the 1st and 2nd order patches. Model C: Combination of the 1st and 3rd patches. Model D: The same as Model C, but the accumulated stress is less except for the centered area.

shots, we show three quantities: rupture time, final slip distribution and maximum slip velocity distribution. Although there is little difference in final slip distribution, the rupture times, namely rupture velocities, are various. In the simplest case (no multi-scalability of Model A), once the rupture is initiated, it accelerates very rapidly and propagates continuously. As expected from our previous studies, rupture velocity is stable and constant in the case where all the scales of patches are superposed (Model B). On the other hand, Models $\mathrm{C}$ and D have multi-scale patches (the 1st and the 3rd scales without the 2 nd one), in which the rupture process is divided into two parts. The rupture initiates on a medium-size patch (the $3 \mathrm{rd}$ scale) and then progresses to the largest (1st scale) patch.

We compare the moment-release-rate functions (sourcetime functions) in Fig. 4. In Model D, we observe a small peak at around $20 \mathrm{~s}$ and the main peak at 70-80 s. This is generally consistent with the results of kinematic slip inversion (e.g. Ide et al., 2011) as shown by a broken line. In this way, it is important that the rupture initiation on the 3rd scale patch seems to be more suitable than the other cases (Models A and B), suggesting that the rupture overcame a big difference of fracture energy up to the large $M 9$ patch. The variation in stress field controls the rup-
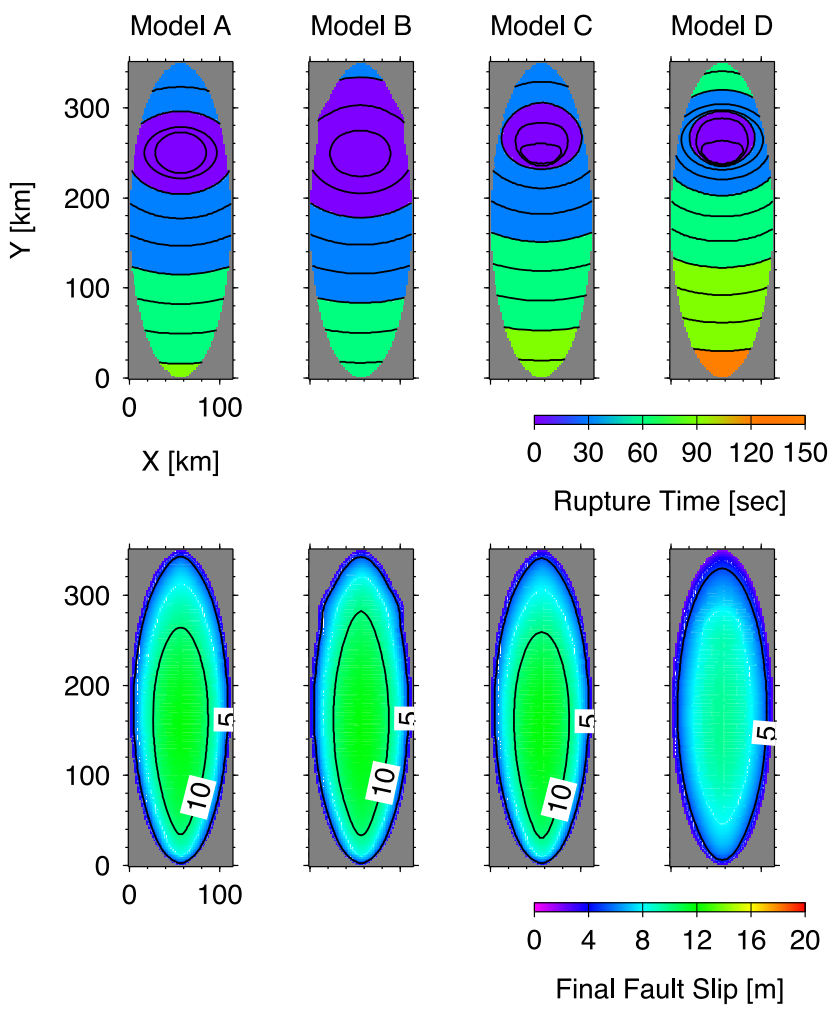

Rupture Time [sec]
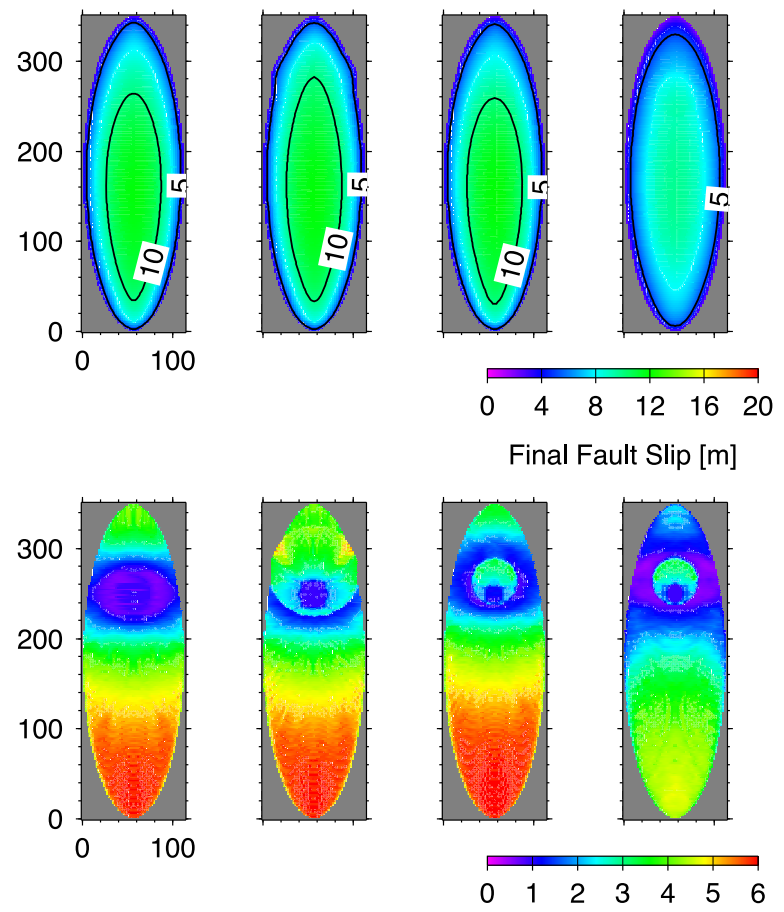

Max. Slip Velocity $[\mathrm{m} / \mathrm{s}]$

Fig. 3. Characterization of dynamic rupture simulation results. Shown for each model is rupture time (contour every $10 \mathrm{~s}$ ), final slip distribution and maximum slip velocity at each point of fault plane. The grey shaded area is not broken.

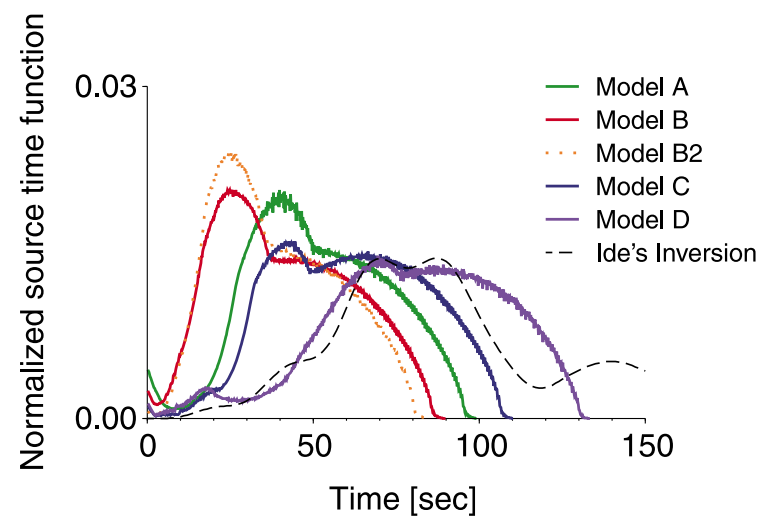

Fig. 4. Source time function normalized by total seismic moment for each model. Model B2 represents the same condition as Model B, except for a stress excess of $5 \mathrm{MPa}$ instead of $6 \mathrm{MPa}$. The magnitudes obtained are all 8.5 except for Model B for which it is 8.6. For reference, the broken line represents the trend obtained by kinematic teleseismic inversion of the Tohoku Earthquake (Ide et al., 2011). 

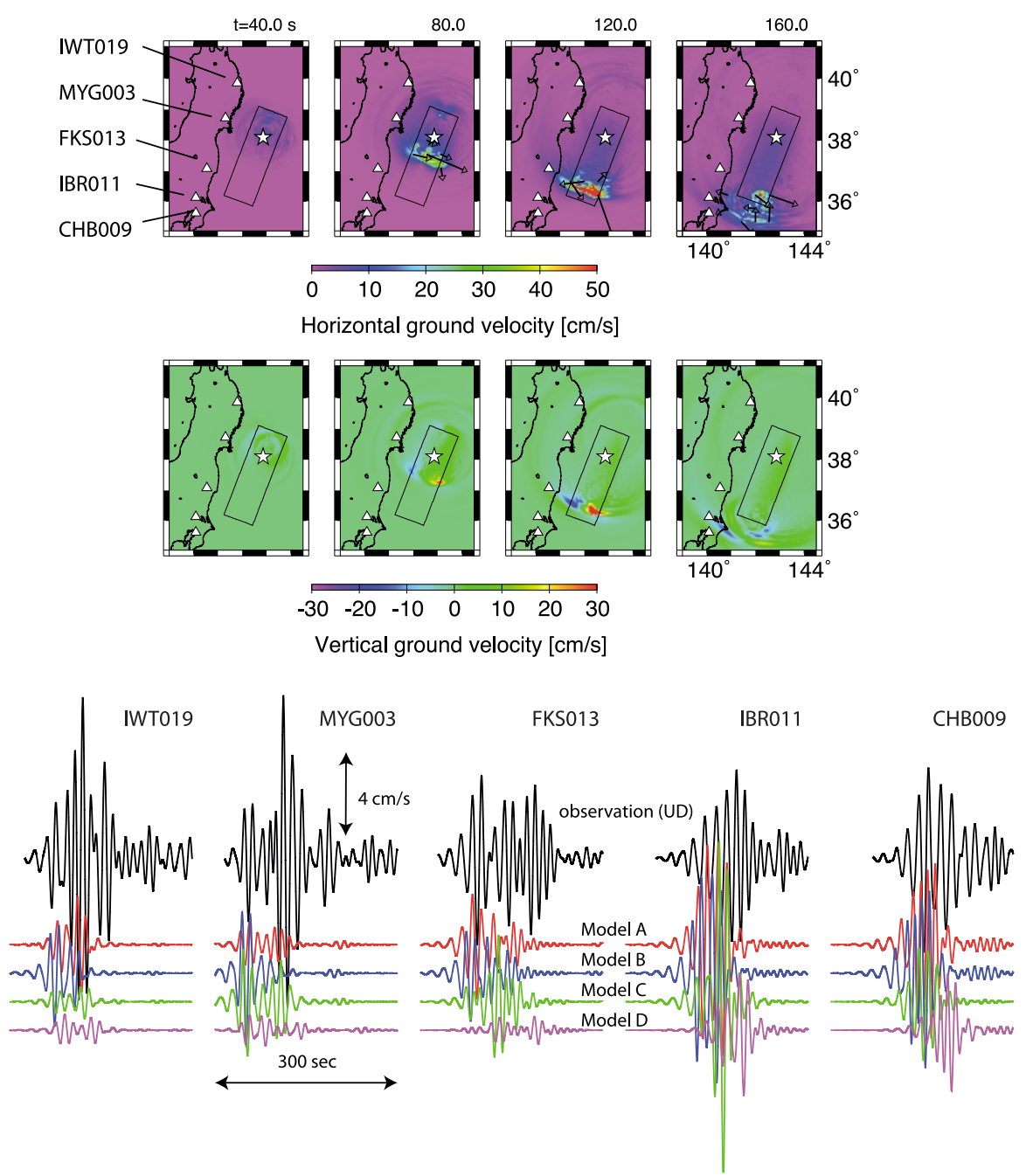

Fig. 5. Snapshots of wave propagation for Model D and a comparison of ground motions at selected stations. The top panel shows horizontal velocity: amplitude in color and direction by arrows. Vertical velocity field is shown in the middle. For each, spatial smoothing is applied every $0.05^{\circ}$, but no temporal filtering is made. At the bottom, the seismograms (vertical ground velocity) are filtered between 10 and $20 \mathrm{~s}$ for all the calculations and observations. The origin time is set at 14:46:18 (JST) after JMA. The signals are shown during $300 \mathrm{~s}$ from the beginning of dynamic rupture onset.

ture velocity, and thus the event duration. For the same patch distribution, a stress excess of $\tau_{\mathrm{p}}=6 \mathrm{MPa}$ (Model B) and $5 \mathrm{MPa}$ (Model B2) are compared. We observe that this variation plays a bigger role after the rupture begins propagating on the largest patch. We then vary the stress field between Models C and D; the latter of which assumes $\tau_{\mathrm{p}}=6.66 \mathrm{MPa}$ on the largest patch instead of the homogeneous condition of $\tau_{\mathrm{p}}=6 \mathrm{MPa}$. The effect is significant in rupture velocity and duration, and produces a trough after the first small peak at around $20 \mathrm{~s}$, which may separate two groups of waves clearly visible in observed strong motion. Such heterogeneity can be likely, as the existence of foreshock activity implies ease of initiating rupture around the hypocenter area.

In our simple system, all the simulations finish with moment magnitudes between 8.5 and 8.6 and a maximum slip of about $10-12 \mathrm{~m}$. These are smaller than that generally reported for this earthquake. In our model, the ruptured area is strictly limited by the largest patch assumed, surrounded by an unbreakable barrier in all directions. However, the dimension of the largest patch is only indicative. The fault area may be surrounded not by a barrier but by an aseismic slip zone or other patches. If the rupture broke the free surface near the trench, the free surface can enhance the rupture near the surface and a much larger fault slip could be expected. It should also be noted that final slip depends on the assumed stress drop, namely twice the stress drop results in twice the slip. Thus obtaining $20 \mathrm{~m}$ of slip and, consequently, a magnitude of 9 is not difficult from the viewpoint of dynamic rupture. However, since our purpose is the qualitative demonstration of rupture growth, we leave the quantitative estimation of fault properties for future work.

\section{Perspective and Conclusion}

We have shown how it could be possible for the Tohoku Earthquake to have been launched and propagated from the point of view of a dynamic rupture process. Although there may have been a long-term preparation in advance, a dynamic process observable in ground motion can be represented by a cascade growth. The transition between two different scale patches is consistent with the two groups of waves we observe in the ground motion. The existence of a larger heterogeneity (having larger $D_{\mathrm{c}}$ ) explains relatively 
uniform and slow rupture propagation over the whole fault length. Our previous model allows the existence of numerous patches, but here we have used the minimum number of patches. This is because we focus on the developing process of dynamic rupture and because small patches do not play significant roles in rupture propagation once the larger patch is broken. However, this could be a necessary modification in order to explain the high-frequency radiation observed in the acceleration. In particular, kinematic inversion indicates another peak in the moment release rate at 140-150 s, and this can originate from a medium-size patch in the south, off shore of Ibaraki.

The main purpose of this paper has been to demonstrate a conceptual dynamic rupture model of the Tohoku Earthquake and it has not been our aim to fit quantitatively the ground motions. However, our model should provide useful information for helping to understand ground motions. We simply calculate the wave propagation using a finite difference method (FDM) (e.g. Aochi and Douglas, 2006, references therein), introducing a 3D deep structure model provided by NIED (Fujiwara et al., 2009). In order to expedite results, we consider a volume of $600 \times 800 \times 50 \mathrm{~km}^{3}$ with a grid spacing of $500 \mathrm{~m}$. Figure 5 shows a snapshot of ground motion for Model D. The first radiation originates from the initial rupture process (until $60 \mathrm{~s}$ ), and then, accompanied by the main rupture propagation, the strongest phase propagates along the whole fault length. Our simple dynamic rupture model is not yet quantitatively embrace the ground motion data in terms of absolute timing and amplitude of phases, as we need further calibration of fault geometries (including the epicenter and the fault plane), and dynamic parameters. However, it is always useful to compare seismograms with observations to investigate further (Fig. 5). As expected from the determined source time functions shown in Fig. 4, Model $\mathrm{B}$ generates a rapidly propagating wave packet, while in Models $\mathrm{C}$ and $\mathrm{D}$, we observe two significant phases around the epicenter latitude (more visibly in Model C at MYG003), the delayed main phase in the south (Ibaraki prefecture), and the long duration of ground motion in the Kanto plain (Chiba prefecture). Model A also leads to two phases, but we should be careful in interpreting this because the rupture is forced to brutally initiate over a large area. The Model A phases may not be suitable as such an initiation process. None of the models is able to explain the large amplitude of the second phase observed around Miyagi and Iwate prefectures and it is important to seek a explanation for this in future studies.

In this paper, we present our considerations soon after the March 11th earthquake. We think that the mechanism of the Tohoku Earthquake is better explained by cascade-rupture growth from a medium size patch to the largest patch rather than by interactions between a few large asperities. Never- theless, we are not sure whether it is appropriate to model the largest scale as a big locked patch. Compared to the kinematic convergence of a plate of $100 \mathrm{~m}$ during 1000 years (plate speed $10 \mathrm{~cm} /$ year), the coseismic the slip of $10-20 \mathrm{~m}$ is still small. It may be expected that the rest of slip is released aseismically. The dynamics of the largest patch might be different from an asperity with $100 \%$ coupling. Anyway, it is regrettable that the largest patch had not been appropriately recognized before the earthquake.

Acknowledgments. The calculations by the BIEM and FDM were carried out at the French national computing center GENCICINES (grant 2011-46700). For the discussion, we used the acceleration data of K-net and KiK-net) from the National Research Institute for Earth Science and Disaster Prevention, Japan. We thank John Douglas, Jeff McGuire, an anonymous reviewer and the editor for improving our manuscript.

\section{References}

Aochi, H. and J. Douglas, Testing the validity of simulated strong ground motion from dynamic rupture of a finite fault, by using empirical equations, Bull. Earthq. Eng., 4, 211-229, 2006.

Aochi, H. and F. Dupros, MPI-OpenMP hybrid simulations using boundary integral equation and finite difference methods for earthquake dynamics and wave propagation: Application to the 2007 Niigata ChuetsuOki earthquake (Mw6.6), International Conference on Computational Science, 2011 (accepted).

Aochi, H. and S. Ide, Numerical study on multi-scaling earthquake rupture, Geophys. Res. Lett., 31(2), doi:10.1029/2003GL018708, 2004.

Aochi, H. and S. Ide, Complexity in earthquake sequences controlled by multi-scale heterogeneity in fault fracture energy, J. Geophys. Res., 114, B03305, doi:10.1029/2008JB006034, 2009.

Di Carli, S., C. François-Holden, S. Peyrat, and R. Madariaga, Dynamic inverion of the 2000 Tottori earthquake based on elliptical subfault approximations, J. Geophys. Res., 115, B12328, doi:10.1029/2009JB006358, 2010.

Fujiwara, H., S. Kawai, S. Aoi, N. Morikawa, S. Senna, N. Kudo, M. Ooi, K. X. Hao, Y. Hayakawa, N. Toyama, H. Matsuyama, K. Iwamoto, H. Suzuki and Y. Liu, A study on subsurface structure model for deep sedimentary layers of Japan for strong-motion evaluation, Tech. Note of National Research Institute for Earth Science and Disaster Prevention, 337, 2009.

Fukuyama, E. and R. Madariaga, Integral equation method for a plane crack with arbitrary shape in 3D elastic media, Bull. Seismol. Soc. Am., 85, 614-628, 1995.

Ide, S. and H. Aochi, Earthquakes as multiscale dynamic ruptures with heterogeneous fracture surface energy, J. Geophys. Res., 110, B11303, doi:10.1029/2004JB003591, 2005.

Ide, S., A. Baltay, and G. C. Beroza, Shallow dynamic overshoot and energetic deep rupture in the $2011 \mathrm{Mw} 9.0$ Tohoku-Oki earthquake, Science, doi:19.1126/science.1207020, 2011.

Madariaga, R. and K. B. Olsen, Criticality of rupture dynamics in 3-D, Pure Appl. Geophys., 157, 1981-2001, 2000.

Peyrat, S., K. B. Olsen, and R. Madariaga, Which dynamic parameters can be estimated from strong ground motion?, Pure Appl. Geophys., 161, 2155-2169, 2004.

Shibazaki, B. and M. Matsu'ura, Spontaneous processes for nucleation, dynamic propagation, and stop of earthquake rupture, Geophys. Res. Lett., 19, 1189-1192, 1992.

H. Aochi (e-mail: h.aochi@brgm.fr) and S. Ide 